\title{
Cerebral Microbleeds: Different Prevalence, Topography, and Risk Factors Depending on Dementia Diagnosis_-The Karolinska Imaging Dementia Study
}

S. Shams, J. Martola, T. Granberg, X. Li, M. Shams, S.M. Fereshtehnejad, L. Cavallin, P. Aspelin, M. Kristoffersen-Wiberg, and L.O. Wahlund

\begin{abstract}
BACKGROUND AND PURPOSE: Cerebral microbleeds are thought to represent cerebral amyloid angiopathy when in lobar regions of the brain and hypertensive arteriopathy when in deep and infratentorial locations. By studying cerebral microbleeds, their topography, and risk factors, we aimed to gain an insight into the vascular and amyloid pathology of dementia diagnoses and increase the understanding of cerebral microbleeds in dementia.
\end{abstract}

MATERIALS AND METHODS: We analyzed 1504 patients ( $53 \%$ women; mean age, $63 \pm 10$ years; 10 different dementia diagnoses) in this study. All patients underwent MR imaging as part of the dementia investigation, and all their clinical parameters were recorded.

RESULTS: Among the 1504 patients with dementia, 22\% had cerebral microbleeds. Cerebral microbleed topography was predominantly lobar $(P=.01)$ and occipital $(P=.007)$ in Alzheimer disease. Patients with cerebral microbleeds were significantly older $(P<.001)$, were more frequently male $(P<.001)$, had lower cognitive scores $(P=.006)$, and more often had hypertension $(P<.001)$. Risk factors for cerebral microbleeds varied depending on the dementia diagnosis. Odds ratios for having cerebral microbleeds increased with the number of risk factors (hypertension, hyperlipidemia, diabetes, male sex, and age 65 and older) in the whole patient group and increased differently in the separate dementia diagnoses.

CONCLUSIONS: Prevalence, topography, and risk factors of cerebral microbleeds vary depending on the dementia diagnosis and reflect the inherent pathology of different dementia diagnoses. Because cerebral microbleeds are seen as possible predictors of intracerebral hemorrhage, their increasing prevalence with an increasing number of risk factors, as shown in our study, may require taking the number of risk factors into account when deciding on anticoagulant therapy in dementia.

ABBREVIATIONS: CAA = cerebral amyloid angiopathy; $C M B=$ cerebral microbleed; ICD = International Classification of Diseases; KIDS $=$ Karolinska Imaging Dementia Study; MMSE = Mini-Mental State Examination

$C^{c}$ erebral microbleeds (CMBs) are not usually seen on conventional MR imaging and CT but have been increasingly detected due to the more frequent use of the $\mathrm{T} 2{ }^{*}$ and SWI MR

Received September 3, 2014; accepted after revision September 16

From the Departments of Neurobiology, Care Sciences, and Society (X.L., S.M.F., L.O.W.) and Clinical Science, Intervention, and Technology (S.S., J.M., T.G., M.S., L.C., P.A., M.K.-W.), Division of Medical Imaging and Technology, Karolinska Institute, Stockholm, Sweden; and Department of Radiology (S.S., J.M., T.G., M.S., L.C., P.A., M.K.-W.) and Division of Clinical Geriatrics (X.L., S.M.F., L.O.W.), Karolinska University Hospital, Stockholm, Sweden.

This work was supported by the Stockholm County Council and Karolinska Institute.

Please address correspondence to Sara Shams, MD, Department of Radiology, Karolinska University Hospital, SE-14186 Stockholm, Sweden; e-mail: sara.shams@ki.se

-- Indicates open access to non-subscribers at www.ajnr.org

$\equiv$ Indicates article with supplemental on-line tables.

Indicates article with supplemental on-line photos.

http://dx.doi.org/10.3174/ajnr.A4176 imaging sequences, sensitive to minute amounts of blood. On MR imaging, CMBs are seen as round hypointense foci, and histologically they are represented by hemosiderin deposits in macrophages, mainly located around small vessels. ${ }^{1,2}$ The pathology of CMBs is thought to vary depending on the location: Deep and infratentorial $\mathrm{CMBs}$ represent underlying hypertensive arteriopathy, whereas lobar CMBs mainly represent vascular amyloid deposition, so-called cerebral amyloid angiopathy (CAA). ${ }^{3}$

CAA and hypertension are common in patients with dementia. CAA is reported to be present in up to $98 \%$ of patients with Alzheimer disease in postmortem studies, and hypertension in middle-aged and elderly populations has been related to the development of dementia. ${ }^{4,5}$ Studies have shown a higher prevalence of CMBs in patients with dementia compared with healthy populations. Alzheimer disease, for instance, is reported to have a CMB prevalence of $18 \%-32 \%$ versus $3 \%-11 \%$ in healthy populations imaged with MR field strengths of $1 \mathrm{~T}-1.5 \mathrm{~T} .^{6-15}$ Consequently, CMBs are hypothesized to play an important role in the 
disease mechanisms of dementia as well as being a marker of the synergistic effects between vascular and amyloid pathology. ${ }^{16}$ Of further interest, CAA and hypertension are the most common causes of intracerebral hemorrhage, with CMBs being proposed as a possible predictor of intracerebral hemorrhage. ${ }^{17}$

Investigating CMBs in dementia is of importance for further understanding the disease mechanisms of different dementia diagnoses and improved clinical and therapeutic treatment. CMBs and their location may give an insight into the vascular and amyloid pathology of dementia diagnoses and thus expose different dementia characteristics. Up-to-date studies on CMBs and dementia have been conducted mainly on small cohorts, without a standardized scale for $\mathrm{CMB}$ rating and with a scarcity of included dementia diagnoses. Furthermore, analyses have been made on a whole-cohort basis, rather than separating different dementia diagnoses and their respective $\mathrm{CMB}$ characteristics. In this study, we aimed to examine the prevalence, topography, and risk factors associated with CMBs in a large and diverse dementia population with subgroup analysis. By doing so, we hoped to gain insight in the pathophysiologic mechanisms in different dementia diagnoses. We hypothesized that CMB prevalence would be dependent on risk factors, depending on the dementia diagnosis, and that vascular risk factors would be important in the development of CMBs in dementia.

\section{MATERIALS AND METHODS \\ Patients}

This study is part of the Karolinska Imaging Dementia Study (KIDS), a cross-sectional study on the impact of CMBs in dementia. Patients undergoing dementia investigation/follow-up from January 1, 2006 to January 1, 2012, and having undergone MR imaging with $\mathrm{CMB}$ sequences were included in this study. A total of 1509 patients were included. Exclusion criteria for all patients were insufficient MR imaging scan quality (3 patients excluded) and a history of traumatic brain injury ( 2 patients excluded), leading to a cohort of 1504 patients.

All patients included had undergone routine dementia assessment, including medical history; physical, neurologic, and cognitive examinations; laboratory tests; and, consequently, MR imaging of the brain with $\mathrm{CMB}$ sequences at the memory clinic/ radiology department, Karolinska University Hospital. Diagnosis was based on the International Classification of Diseases (ICD)-10 criteria, by an experienced memory clinic team consisting of geriatricians, neuropsychologists, neurophysiologists, and neuroradiologists after the whole picture had been considered. All patients were subdivided into 10 diagnostic groups on the basis of the ICD-10 codes, shown in On-line Table 1 . If $>1$ ICD code existed for a patient, the current ICD code at the dementia investigation/MR imaging scan was chosen.

Clinical data were collected for every patient during the dementia investigation. The presence of hypertension, hyperlipidemia, and diabetes was determined on the basis of prior medical diagnosis and treatment for all patients. Vascular risk factors are included for all patients in this study, and the Mini-Mental State Examination (MMSE) scores, for 1416 patients. The percentage of MMSE scores in the diagnoses was $91 \%-97 \%$, except in frontotemporal lobe dementia (77\%) and asymptomatic hereditary dementia (73\%). The MMSE score was missing due to the following: patient too severely disoriented to participate $(6 \%, n=5$; Alzheimer disease and vascular dementia only) and score registration missing in the patient notes, even though it was performed (94\%, $n=83$ ). Informed consent was obtained from each patient; if the patient was too confused to consent, it was obtained from a legal guardian. Ethics approval was obtained from the regional ethics board, Stockholm, Sweden.

\section{MR Imaging Protocols}

All patients underwent MR imaging of the brain at the radiology department, Karolinska University Hospital, Huddinge, Sweden. For each patient, axial SWI and/or $\mathrm{T} 2{ }^{\star}$ sequences and conventional MR imaging sequences, such as T1, T2, and FLAIR, were performed. Three MR imaging scanners at the radiology department, Karolinska University Hospital, Huddinge, Sweden, were used. Of all patients, 453 patients were scanned on a 1.5T Magnetom Symphony scanner (Siemens, Erlangen, Germany; T2*: TE, $25 \mathrm{~ms}$; TR, $792 \mathrm{~ms}$; flip angle, $20^{\circ}$; section thickness, $5.0 \mathrm{~mm}$ ), 687 patients were scanned on a 1.5T Magnetom Avanto scanner (Siemens; T2*: TE, $26 \mathrm{~ms}$; TR, $800 \mathrm{~ms}$; flip angle, $20^{\circ}$; section thickness, $5.0 \mathrm{~mm}$; SWI: TE, $40 \mathrm{~ms}$; TR, $49 \mathrm{~ms}$; flip angle, $15^{\circ}$; section thickness, $4.0 \mathrm{~mm}$ ), and 364 patients were scanned on a Magnetom Trio scanner (Siemens; 3T; T2*: TE, $20 \mathrm{~ms}$; TR, $620 \mathrm{~ms}$; flip angle, $20^{\circ}$; section thickness, $4.0 \mathrm{~mm}$; SWI: TE, $20 \mathrm{~ms}$; TR, $28 \mathrm{~ms}$; flip angle, $15^{\circ}$; section thickness, $1.6 \mathrm{~mm}$ ). Scans of all patients were randomly assigned to various $\mathrm{MR}$ imaging scanners with $1.5 \mathrm{~T}$ and $3 \mathrm{~T}$ field strengths and different $\mathrm{CMB}$ sequences, $\mathrm{T} 2^{*}$ and SWI. In the whole cohort, the distribution of patients scanned at 3T with SWI sequences included is seen in On-line Table 1.

\section{Image Analysis}

All MR images first underwent routine analysis by the neuroradiologists at the radiology department, Karolinska University Hospital. In addition, all MR images were analyzed specifically for CMBs by an $\mathrm{MD} / \mathrm{PhD}$ student, with 2 years of training and experience in MR imaging and neuroradiology, at the initial time of rating. The rater was blinded to clinical data and assessed the number and location of CMBs on $\mathrm{T}^{*}$ and/or SWI sequences according to the Microbleed Anatomical Rating Scale. ${ }^{2}$ Minor modifications were made to the Microbleed Anatomical Rating Scale to increase the accuracy of CMB ratings: $\mathrm{CMBs}$ were not rated as probable; hypointensities in the globus pallidus were not rated to reduce the risk of calcifications and physiologic iron deposition mimicking CMBs. Furthermore, if a patient had a deep venous anomaly in the vicinity of a $\mathrm{CMB}$, the $\mathrm{CMB}$ was not rated as definitive because deep venous anomalies increase the risk of adjacent cavernomas that, in turn, can mimic a CMB. $\mathrm{T} 2$-weighted images were analyzed simultaneously with the $\mathrm{CMB}$ sequences, to better distinguish vessels and flow voids, which might mimic CMBs.

To ensure a correct $\mathrm{CMB}$ rating, a senior consultant neuroradiologist rated 50 patients with $\mathrm{CMBs}$ with wide distributions in different locations. The 50 patients with CMBs were selected from the KIDS database established after analysis by choosing the first 10 patients with CMBs for every year from 2006 to 2010, to get a thorough representation of CMBs analyzed. Besides the 50 patients with CMBs, 50 patients without CMBs were also analyzed, 
all chosen in the same manner as patients with CMBs. The second rater was blinded to clinical data and prior ratings. Interrater analysis yielded an intraclass correlation coefficient of 0.988 when the 50 patients with CMBs were taken into account and 0.987 when all analyses were considered. Both coefficients equal an excellent agreement. ${ }^{18}$ Intrarater analysis was also performed by the MD/ $\mathrm{PhD}$ student, rating the whole cohort for CMBs in the same manner as above (blinded to clinical data, prior ratings). Two months after the initial analysis, the first 100 patients with CMBs and the first 100 patients without CMBs were re-rated. Intraclass correlation analysis showed excellent agreement, $0.989(n=200)$, and 0.991 for the 100 patients with CMBs only. All neuroradiologic analyses were made on a radiologic PACS workstation with 3 radiologic monitors.

\section{Statistical Analysis}

Descriptive statistics are presented as means $( \pm S D)$ for continuous variables and median and interquartile range for CMBs. Inter- and intrarater analysis was performed with the intraclass correlation coefficient on the number of CMBs and is presented in "Image Analysis." Differences in population characteristics and CMBs in the diagnoses were analyzed by using $\chi^{2}$ and Fisher exact tests for categoric variables. CMBs were defined as dichotomous (present/absent). Due to our nonparametric data, the Mann Whitney $U$ test was used for continuous variables. Kruskall-Wallis analysis was performed when analyzing CMB topography in the cohort, with topographies as categoric variables and CMBs as a continuous variable. The topographic analysis was performed in the 3 Microbleed Anatomical Rating Scale locations and more specific infratentorial and lobar locations. Logistic regression was performed for all diagnostic groups with CMBs (dichotomous variable: present/absent) as a dependent variable and diagnosis as an independent variable. For each diagnosis, the subjective cognitive impairment was set as a reference group. Odds ratios were adjusted for hypertension, hyperlipidemia, diabetes, sex, age, MR imaging field strength $(1.5 / 3 \mathrm{~T})$, and CMB sequence (SWI/T2*). Logistic regression was further used to obtain odds ratios for CMBs, depending on the number of risk factors. CMB (dichotomous: present/absent) was a dependent variable, with the number of risk factors as an independent variable. The model was adjusted for MR imaging field strength and CMB sequence. Negative binomial regression analyses were used to determine the impact of the number of CMBs/number of CMBs in different topographies on risk factors. The number of CMBs was a dependent variable, and risk factor, an independent variable; the model was corrected for MR imaging field strength and CMB sequence. Post hoc Bonferroni correction was applied to all $P$ values; thus, all significance levels presented in this article are Bonferroni-corrected, unless otherwise stated. SPSS 22.0 (IBM, Armonk, New York) was used for statistical analyses, and $P<.05$ was statistically significant.

\section{RESULTS}

\section{Prevalence}

In the 1504 recruited patients, 332 (22\%) had CMBs. Patient characteristics are shown in On-line Table 2. The prevalence $\left(\chi^{2}=94 ; P<.001\right)$ and number $(P<.001)$ of CMBs and the presence of multiple $\left(\chi^{2}=25 ; P=.03\right) \mathrm{CMBs}$ varied significantly among the different diagnostic groups (On-line Table 2 and Online Fig 1). The significant adjusted odds ratios for having CMBs in the different diagnoses were the following: alcohol-related dementia (OR, 4.0; 95\% CI, 1.4-11.2), Alzheimer disease (OR, 2.0; 95\% CI, 1.2-3.1), unspecified dementia (OR, 2.2; 95\% CI, 1.04.4 ), and vascular dementia (OR, 8.7; 95\% CI, 4.1-18.6) (On-line Table 2).

\section{Topography}

The most common CMB topography among all patients with CMBs $(n=332)$ was lobar; this was also true in the separate groups (On-line Table 3). Eighty-four percent had CMBs in lobar regions, followed by infratentorial topography in $30 \%$ of patients and deep topography in 27\% (On-line Table 3). In patients with CMBs ( $n=332$ ), 487 CMBs (median, 1 ; interquartile range, $1-5$ ), were found in infratentorial locations; 367 , in deep locations (median, 2; interquartile range, 1-3); and 2415 CMBs, in lobar locations (median, 2; interquartile range, 1-5). Kruskall-Wallis analysis of the topographic distribution of CMBs, for all diagnoses, in infratentorial, deep, and lobar regions showed a significant lobar predominance of CMBs only in Alzheimer disease $(P=.01)$. Further analysis for more detailed brain regions (brain stem; cerebellum; deep; frontal, parietal, temporal, and occipital lobes) in the diagnoses showed a significant difference among these locations only in Alzheimer disease, with most CMBs in occipital regions $(P=.007)$.

\section{Risk Factors and Associations}

Comparing patients with $(n=332)$ and without CMBs $(n=$ 1172 ), we found that CMBs were significantly more frequent in male patients $\left(\chi^{2}=37 ; P<.001\right)$, older patients $\left(\chi^{2}=32 ; P<\right.$ $.001)$, and those with hypertension $\left(\chi^{2}=23 ; P<.001\right)$ (On-line Table 4). There was no significant association between CMBs and diabetes, hyperlipidemia, and low MMSE score $(<21)$ in the whole cohort. Risk factors and association with CMBs for the separate groups are shown in On-line Table 4. Mann Whitney $U$ analysis for the whole cohort showed lower MMSE scores with CMBs (mean MMSE score: $\mathrm{CMB}+, 24 \pm 5$; $\mathrm{CMB}-, 25 \pm 5 ; P=.02)$. In the separate groups, this was only true in unspecified dementia $(\mathrm{CMB}+$, $24 \pm 5 ; \mathrm{CMB}-, 21 \pm 5 ; P=.01)$ and asymptomatic hereditary dementia (mean MMSE score: $\mathrm{CMB}+, 28 \pm 1 ; \mathrm{CMB}-, 29 \pm 1 ; P=$ .032) before Bonferroni correction, and it was insignificant after correction.

In the separate groups, $\mathrm{CMB}$ prevalence was higher with hypertension in subjective cognitive impairment $\left(\chi^{2}=8 ; P=.02\right)$ and higher with hyperlipidemia in mild cognitive impairment $\left(\chi^{2}=7 ; P=.03\right)$. Male patients further had a higher prevalence of CMBs in Alzheimer disease $\left(\chi^{2}=8 ; P=.02\right)$, mild cognitive impairment $\left(\chi^{2}=10 ; P=.01\right)$, and vascular dementia $\left(\chi^{2}=7\right.$; $P=.04)$. Older patients, defined as 65 years and older, had a higher CMB prevalence in Alzheimer disease $\left(\chi^{2}=9 ; P=.01\right)$ and mild cognitive impairment $\left(\chi^{2}=8 ; P=.02\right)$ (On-line Table 4$)$.

When we took the number of CMBs and the relation to risk factors into account, in a multivariate negative binomial regression analysis, male sex, advanced age, and hypertension showed an increasing number of CMBs in the whole cohort. A decreasing number of CMBs with hyperlipidemia and diabetes were shown. 
Prevalence and odds ratios of CMBs depending on number of risk factors ${ }^{\mathrm{a}}$

\begin{tabular}{|c|c|c|c|c|c|c|c|c|c|}
\hline \multirow[b]{2}{*}{ Diagnostic Group } & \multirow{2}{*}{$\begin{array}{l}\mathrm{ORF}^{\mathrm{b}} \\
\text { Prevalence }\end{array}$} & \multicolumn{2}{|c|}{$1 \mathrm{RF}$} & \multicolumn{2}{|c|}{2 RF } & \multicolumn{2}{|c|}{$3 \mathrm{RF}$} & \multicolumn{2}{|c|}{$\geq 4 \mathrm{RF}$} \\
\hline & & Prevalence & OR & Prevalence & OR & Prevalence & OR & Prevalence & OR \\
\hline All patients $(n=1504)$ & $10(31)$ & $18(92)$ & $2.1(1.4-3.3)^{\mathrm{d}}$ & $28(105)$ & $3.8(2.5-5.9)^{c}$ & $35(69)$ & $5.3(3.3-8.5)^{c}$ & $33(35)$ & $4.8(2.8-8.4)^{c}$ \\
\hline $\begin{array}{l}\text { Subjective cognitive impairment } \\
\quad(n=385)\end{array}$ & $8(13)$ & $10(13)$ & $1.2(0.5-2.8)$ & $11(6)$ & $1.3(0.5-3.8)$ & $24(7)$ & $3.6(1.3-10.2)^{e}$ & $20(2)$ & $3.2(0.6-17.2)$ \\
\hline Alzheimer disease $(n=423)$ & $7(4)$ & $26(37)$ & $4.9(1.7-14.6)^{d}$ & & $8.2(2.8-24.4)^{\mathrm{c}}$ & $33(23)$ & $7.1(2.3-22.2)^{\mathrm{d}}$ & $23(7)$ & $3.4(0.9-13.1)$ \\
\hline Mild cognitive impairment $(n=418)$ & $7(5)$ & $17(24)$ & $2.8(1.0-7.7)^{e}$ & $22(25)$ & $3.7(1.3-10.3)^{e}$ & $37(21)$ & $7.8(2.7-22.9)^{\mathrm{c}}$ & 35 (14) & $7.2(2.2-21.3)^{d}$ \\
\hline Vascular dementia $(n=54)$ & $67(4)$ & $50(3)$ & $0.5(0.04-6.1)$ & $58(11)$ & $0.6(0.1-4.7)$ & $58(7)$ & $0.5(0.1-4.8)$ & $64(7)$ & $0.8(0.1-7.1)$ \\
\hline
\end{tabular}

Note:-RF indicates risk factors; OR, odds ratio of CMBs in all groups; Prevalence, prevalence of CMBs in all groups.

${ }^{a}$ Numbers are \% (No.), odds ratio $(95 \% \mathrm{Cl})$. Logistic regression analysis was performed with CMBs (present/absent) as a dependent variable and number of risk factors as an independent variable. Risk factors were defined as hypertension, hyperlipidemia, diabetes, male sex, and age 65 years and older. The model was corrected for MRI field strength $(1.5 \mathrm{~T} / 3 \mathrm{~T})$ and $\mathrm{CMB}$ sequence $(\mathrm{T} 2 * / \mathrm{SWI})$. In the risk factor groups, prevalence of CMBs (number of patients) is stated to the left and odds ratios for CMBs, to the right.

${ }^{b}$ Patients with zero risk factors were used as a reference.

${ }^{c} P<.001$, significant after Bonferroni correction.

d $p<.05$, significant after Bonferroni correction.

e $P<.05$, significant before Bonferroni correction.

On-line Table 5 shows the regression coefficients for the risk factors in the 4 largest diagnoses.

$\mathrm{CMB}$ prevalence and odds ratio for the presence of CMBs increased with the increasing number of risk factors in the whole patient group (Table). The Table shows the impact of the number of risk factors on $\mathrm{CMB}$ prevalence and odds ratios in the 4 largest diagnostic groups. On-line Fig 2 illustrates the increase in odds ratios for number of risk factors in the whole patient group.

The topography of CMBs and the association to risk factors were analyzed. Patients with CMBs in deep and infratentorial locations were compared with patients with CMBs in lobar regions regarding patient characteristics (hyperlipidemia, diabetes, sex, age, and MMSE score). The only difference found between the 2 groups was $62 \%$ of all patients with deep and infratentorial CMBs having hypertension, compared with $48 \%$ in patients with lobar CMBs $\left(\chi^{2}=10 ; P=.006\right)$. When we took the number of CMBs into account in a multivariate negative binomial regression analysis, CMBs were associated with different risk factors in the different topographies and diagnoses (On-line Table 5).

\section{DISCUSSION}

Our study provides insight in CMBs and the relation to various dementia diagnoses. Prevalence, topography, and risk factors (hypertension, hyperlipidemia, diabetes, male sex, and older age) for $\mathrm{CMBs}$ vary depending on the dementia diagnosis. Prevalence and odds ratios for $\mathrm{CMBs}$ increase with the increasing number of risk factors in the whole patient group and increase differently in different dementia diagnoses.

To the best of our knowledge, this is the first study on CMBs and dementia in a large and diverse cohort with subgroup analysis and emphasis on risk factors for the presence of CMBs. The prevalence of CMBs detected in our study is in line with previously published results. ${ }^{6-8,13-15,19,20}$ However, the mean age in other studies has generally been higher, therefore presumably occasionally leading to a higher CMB prevalence. Similarly, studies using higher field strengths and the SWI sequence have shown a higher prevalence of CMBs. $^{21}$

Our findings may have several important clinical implications. The association of lobar CMBs with CAA and deep CMBs with hypertensive arteriopathy has been shown and discovered in pathologic and risk factor analysis studies and further by in vivo PET imaging with Pittsburgh compound-B. ${ }^{22-29}$ The prevalence, topography, and risk factors of CMBs may thus give an insight in the vascular and amyloid components of different dementia diagnoses.

Our results showed a significantly predominant lobar and occipital $\mathrm{CMB}$ topography in Alzheimer disease, typical for $\mathrm{CAA},{ }^{30-33}$ reflecting the importance of amyloid pathology in $\mathrm{Alz}-$ heimer disease. Lobar topography of CMBs in Alzheimer disease has been reported before, ${ }^{6,8,13,19,20}$ with occipital predominance only reported in 1 study. ${ }^{6}$ The $\mathrm{CMB}$ topography in the whole cohort was predominantly lobar, with a large amount of strictly lobar CMBs compared with CMBs strictly in infratentorial and deep regions. This may reflect the predominance of CAA and further amyloid pathology in dementia, as well as increasing CAA with age. Our topographic analysis further showed CMBs in both lobar and deep and infratentorial locations across dementia diagnoses, with a relation to hypertension in both topographies, corroborating the synergistic/additive effects of amyloid and vascular disease in dementia.

The concomitant effects between amyloid and vascular pathology are further suggested in our results. Hypertension and hyperlipidemia were both accompanied by higher CMB prevalence in the whole patient group and in mild and subjective cognitive impairment. Furthermore, hypertension in subjective cognitive impairment and hyperlipidemia in mild cognitive impairment were significantly associated with an increasing number of CMBs. The association of CMBs with hypertension and hyperlipidemia in the early dementia stages may suggest a stepwise process, with vascular risk factors important early in the dementia process, later overtaken by amyloid pathology. Likewise, theories exist that hypertension may be an important risk factor for the development of Alzheimer disease. ${ }^{5}$ The odds ratios for CMBs, adjusted for vascular risk factors, sex, and advanced age support this theory. Only alcohol-related dementia, Alzheimer disease, unspecified dementia, and vascular dementia had significant odds ratios for developing CMBs, when adjusting for vascular risk factors. This finding suggests the importance of amyloid pathology in the development of CMBs in Alzheimer disease, vascular dementia, and possibly also in alcohol-related dementia, though unreported cerebral contusions might be possible in the latter case as well.

Prevalence and number of CMBs rendered a slightly different risk factor profile across the diagnoses. In the whole cohort, the prevalence of CMBs increased with hypertension and hyperlipid- 
emia, and the number of CMBs decreased with hyperlipidemia and diabetes. The decrease in CMBs with hyperlipidemia may imply that hyperlipidemia in itself merely causes or leads to the development of CMBs, without major impact on the number of CMBs. The association of diabetes with a decreasing number of CMBs and the lack of association with the prevalence of CMBs suggest that diabetes in itself may not be a strong risk factor for CMBs in dementia. Hypertension, male sex, and advanced age were associated with consistently higher prevalence/number of CMBs and are thus presumably strong risk factors for CMBs across dementia diagnoses. The MMSE score increased with an increasing number of CMBs in Alzheimer disease and mild cognitive impairment, suggesting that the number of CMBs does not directly impact cognition.

Another potentially important clinical implication of our study is the increasing $\mathrm{CMB}$ prevalence with number of risk factors. Odds ratios for $\mathrm{CMBs}$ increased with the increasing number of risk factors (vascular risk factors, male sex, and older age) in the whole patient group. Because CMBs have been suggested as predictors of intracerebral hemorrhage, the number of risk factors may be an important factor to consider, especially in patients with anticoagulant medication. However, more research is warranted to outline the role of CMBs as a predictor of intracerebral hemorrhage and thus the clinical implications of our finding.

Our study has several strengths. A large and diverse cohort, subgroup analysis, and the use of a standardized scale for CMB rating accompanied by excellent inter- and intrarater agreement are major strengths, contributing to the generalizability of our study. Limitations, on the other hand, encompass the use of different MR imaging parameters and field strengths. Nevertheless, patients were randomly assigned to different scanners, with almost equal representation of MR imaging field strength/CMB sequence across diagnoses. Furthermore, we corrected all our regression models for the potential effect of MR imaging field strength and $\mathrm{CMB}$ sequence.

\section{CONCLUSIONS}

Our study demonstrates the variance of CMBs in dementia, providing an insight into different dementia diagnoses. Future studies in the KIDS will focus on CSF markers in relation to CMBs and further neuroradiologic interpretations.

\section{ACKNOWLEDGMENTS}

We thank Helena Forssell and Karin Kjellsdotter for their administrative and technical assistance.

\section{REFERENCES}

1. Fazekas F, Kleinert R, Roob G, et al. Histopathologic analysis of foci of signal loss on gradient-echo $\mathrm{T} 2^{\star}$-weighted MR images in patients with spontaneous intracerebral hemorrhage: evidence of microangiopathy-related microbleeds. AJNR Am J Neuroradiol 1999;20: 637-42

2. Gregoire SM, Chaudhary UJ, Brown MM, et al. The Microbleed Anatomical Rating Scale (MARS): reliability of a tool to map brain microbleeds. Neurology 2009;73:1759-66

3. Auer RN, Sutherland GR. Primary intracerebral hemorrhage: pathophysiology. Can J Neurol Sci 2005;32(suppl 2):S3-12

4. Jellinger KA. Alzheimer disease and cerebrovascular pathology: an update. J Neural Transm 2002:109:813-36
5. Skoog I. The relationship between blood pressure and dementia: a review. Biomed Pharmacother 1997;51:367-75

6. Pettersen JA, Sathiyamoorthy G, Gao FQ, et al. Microbleed topography, leukoaraiosis, and cognition in probable Alzheimer disease from the Sunnybrook dementia study. Arch Neurol 2008;65:790-95

7. Nakata-Kudo Y, Mizuno T, Yamada K, et al. Microbleeds in Alzheimer disease are more related to cerebral amyloid angiopathy than cerebrovascular disease. Dement Geriatr Cogn Disord 2006;22:8-14

8. Cordonnier C, van der Flier WM, Sluimer JD, et al. Prevalence and severity of microbleeds in a memory clinic setting. Neurology 2006;66:1356-60

9. Roob G, Schmidt R, Kapeller P, et al. MRI evidence of past cerebral microbleeds in a healthy elderly population. Neurology 1999;52: 991-94

10. Jeerakathil T, Wolf PA, Beiser A, et al. Cerebral microbleeds: prevalence and associations with cardiovascular risk factors in the Framingham Study. Stroke 2004;35:1831-35

11. Sveinbjornsdottir S, Sigurdsson S, Aspelund T, et al. Cerebral microbleeds in the population based AGES-Reykjavik study: prevalence and location. J Neurol Neurosurg Psychiatry 2008;79: 1002-06

12. Tsushima Y, Tanizaki Y, Aoki J, et al. MR detection of microhemorrhages in neurologically healthy adults. Neuroradiology 2002;44: 31-36

13. Hanyu H, Tanaka Y, Shimizu S, et al. Cerebral microbleeds in Alzheimer's disease. J Neurol 2003;250:1496-97

14. Nakata Y, Shiga K, Yoshikawa K, et al. Subclinical brain hemorrhages in Alzheimer's disease: evaluation by magnetic resonance T2* -weighted images. Ann N Y Acad Sci 2002;977:169-72

15. Fukui T, Oowan Y, Yamazaki T, et al. Prevalence and clinical implication of microbleeds in dementia with Lewy bodies in comparison with microbleeds in Alzheimer's disease. Dement Geriatr Cogn Dis Extra 2013;3:148-60

16. Cordonnier C, van der Flier WM. Brain microbleeds and Alzheimer's disease: innocent observation or key player? Brain 2011: 134(pt 2):335-44

17. Lee $\mathrm{SH}, \mathrm{Bae} \mathrm{HJ}$, Kwon SJ, et al. Cerebral microbleeds are regionally associated with intracerebral hemorrhage. Neurology 2004;62: 72-76

18. Landis JR, Koch GG. The measurement of observer agreement for categorical data. Biometrics 1977;33:159-74

19. Uetani H, Hirai T, Hashimoto M, et al. Prevalence and topography of small hypointense foci suggesting microbleeds on $3 \mathrm{~T}$ susceptibility-weighted imaging in various types of dementia. AJNR Am J Neuroradiol 2013;34:984-89

20. Brundel M, Heringa SM, de Bresser J, et al. High prevalence of cerebral microbleeds at 7Tesla MRI in patients with early Alzheimer's disease. J Alzheimers Dis 2012;31:259-63

21. Nandigam RNK, Viswanathan A, Delgado P, et al. MR imaging detection of cerebral microbleeds: effect of susceptibility-weighted imaging, section thickness, and field strength. AJNR Am J Neuroradiol 2009;30:338-43

22. Dierksen GA, Skehan ME, Khan MA, et al. Spatial relation between microbleeds and amyloid deposits in amyloid angiopathy. Ann Neurol 2010;68:545-48

23. Park JH, Seo SW, Kim C, et al. Pathogenesis of cerebral microbleeds: in vivo imaging of amyloid and subcortical ischemic small vessel disease in 226 individuals with cognitive impairment. Ann Neurol 2013;73:584-93

24. Gurol ME, Dierksen G, Betensky R, et al. Predicting sites of new hemorrhage with amyloid imaging in cerebral amyloid angiopathy. Neurology 2012;79:320-26

25. Greenberg SM, Vernooij MW, Cordonnier C, et al. Cerebral microbleeds: a guide to detection and interpretation. Lancet Neurol 2009;8:165-74

AJNR Am J Neuroradiol 36:661-66 Apr 2015 www.ajnr.org 665 
26. Werring DJ, ed. Cerebral Microbleeds: Pathophysiology to Clinical Practice. Cambridge: Cambridge University Press; 2011

27. Poels MM, Vernooij MW, Ikram MA, et al. Prevalence and risk factors of cerebral microbleeds: an update of the Rotterdam scan study. Stroke 2010:41(10 suppl):S103-06

28. Wiegman AF, Meier IB, Schupf N, et al. Cerebral microbleeds in a multiethnic elderly community: demographic and clinical correlates. J Neurol Sci 2014;345:125-30

29. Romero JR, Preis SR, Beiser A, et al. Risk factors, stroke prevention treatments, and prevalence of cerebral microbleeds in the Framingham Heart Study. Stroke 2014;45:1492-94
30. Vinters HV, Gilbert JJ. Cerebral amyloid angiopathy: incidence and complications in the aging brain. II. The distribution of amyloid vascular changes. Stroke 1983;14:924-28

31. Biffi A, Greenberg SM. Cerebral amyloid angiopathy: a systematic review. J Clin Neurol 2011;7:1-9

32. Rosand J, Muzikansky A, Kumar A, et al. Spatial clustering of hemorrhages in probable cerebral amyloid angiopathy. Ann Neurol 2005;58:459-62

33. Attems J, Jellinger KA, Lintner F. Alzheimer's disease pathology influences severity and topographical distribution of cerebral amyloid angiopathy. Acta Neuropathol 2005;110:222-31 